\title{
Erratum: Field Theory of Critical Behavior in a Driven Diffusive System ${ }^{1}$
}

\author{
Kwan-tai Leung ${ }^{2}$ and John L. Cardy ${ }^{2}$
}

Received August 11, 1986

It has been pointed out that there is a sign error in (A.3) which leads to the absence of a nontrivial fixed point in the case $r_{\|}=0, r_{\perp}>0$, and that the cancellation mechanism in Appendix III is incomplete. The correct version will appear in a follow-up paper to Ref. 4 (Janssen and Schmittmann, $Z$. Phys. B, to appear). We thank those who brought these errors to our attention. However, we emphasize that our main results, which refer to $r_{\perp}=0$, $r_{\|}>0$, remain valid.

\footnotetext{
${ }^{1}$ This paper appeared in J. Stat. Phys. 44:567 (1986).

${ }^{2}$ Department of Physics, University of California, Santa Barbara, California 93106.
} 